\title{
"Formación" Simití: unidad ambigua en la estratigrafía del Valle Medio del Magdalena
}

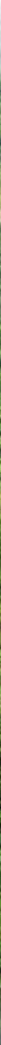

We come therefore to the problem of defining the individual stratigraphical unit, which is the basis of nearly all our troubles 
Portadilla: Prístina localidad tipo de la "Tablazo Pasture Formation" de Wheeler (1929), hoy en día "Formación" Simití. La textura de fondo de la página corresponde a la sección delgada de una capa arenoso-glauconítica con cemento calcáreo.

$\mathrm{N}: 1.269 .864$ E:1.081.171 Z:358 azimut $26^{\circ}$

Citación: Etayo-Serna, Fernando. 2019. "'Formación' Simití: unidad ambigua en la estratigrafía del Valle Medio del Magdalena". En Estudios geológicos y paleontológicos sobre el Cretácico en la región del embalse del río Sogamoso, Valle Medio del Magdalena, dirección científica y edición de Fernando Etayo-Serna. Compilación de los Estudios Geológicos Oficiales en Colombia vol. XXIII. Bogotá: Servicio Geológico Colombiano.

\section{$A \quad b \quad s \quad t \quad r \quad a \quad c \quad t$}

In the Hidrosogamoso region, Wheeler's "Tablazo Pasture formation" became the Simití Formation of Morales et al. It consists of dark, muscovitic and fissile mudstones (shales), with abundant 'small irregular shaped nodules and concretions of limonite and hematite', interspersed throughout with fine-grained quartzitic sandstones that are more often than not glauconitic. In this region, Simití represents the lower Middle Albian; however, towards the NW Ciénaga Simití, it ranges from the Lower Albian to the lower Upper Albian. This stratigraphic record suggests that the east margin of the MMV was exposed, while sedimentation was continuous in the Simiti region up to the early late Albian, developing an asymmetrically thick sedimentary sequence, indicating that deposition of the Simití was controlled by regional tectonism and erosion prior to deposition of the Cenomanian El Salto Formation. 


\section{"Formación" Simití: unidad ambigua en la estratigrafía del Valle Medio del Magdalena}

Fernando Etayo-Serna

El comité ad hoc conformado por la Industria del Petróleo de Colombia para estandarizar la nomenclatura estratigráfica aplicable a las unidades litológicas cretácicas del Valle Medio del Magdalena (VMM) presentó un esquema que incluyó denominaciones formales e informales (Salvador 1994), como es el caso del Simití Shale (fig. 5, en Morales y The Colombian Petroleum Industry 1958). Se deben a Enrique Hubach las combinaciones nomenclaturales "Formación Simiti" y "Formación de Simití (Simití Shales)" (Hubach 1957, 137 e índice). La presente nota llama la atención sobre la falta de precisión asociada al uso de esta terminología.

Morales y The Colombian Petroleum Industry (1958, 651, fig 5) adoptaron el nombre "Simiti Shale [...] to designate the excellent outcrops of this part of the section exposed along the south bank of the Cienaga Simití (sic)". Además señalaron que "Its relationship with the underlying Tablazo limestone is conformable and the contact is relatively sharp" (op. cit., 652).

En el costado suroriental (SE) del VMM, la llamada "Formación" Simití reposa sobre la Formación Tablazo (Montoya 2019). Estudios geológicos en la región de la ciénaga de Simití, costado noroccidental (NW) del VMM, muestran que la "Formación" Simití yace sobre la Formación La Paja a manera de continuación de esta. Su contacto superior, tanto en la región de Simití como en la de Hidrosogamoso (SE) se sitúa por debajo de las calizas con Exogyra que componen la Formación El Salto (Ingeominas-Universidad Industrial de Santander 2006).

La expresión "Formación" Simití, como se utiliza hoy en día en la región del río Sogamoso (Ward et al. 1973), corresponde a la Tablazo Pasture Formation propuesta por Wheeler en 1929 (Morales y The Colombian Petroleum Industry 1958, 652). En el sector del VMM revisado en el presente estudio tiene su mejor expresión morfológica en la margen $\mathrm{N}$ del río Sogamoso, frente a la antigua casa El Tablazo, situada en el cuadrante SE de la plancha topográfica 120-I-B del IGAC, plancha geológica del Ingeominas H-12 (cuadrícula B4 1/4 SE) y en el mapa geológico de Moreno-Sánchez (2019) (D4 1/4 SE). Dicho sitio fue el escogido por Wheleer (1929) como localidad tipo de su Tablazo Pasture Formation, la que describió como sigue:

Resting upon the Tablazo formation is a thick deposit of black shales about 500 meters (1640') thick that are called the Tablazo Pasture Shales. This discordant term was applied to these beds since they are typically exposed in Tablazo Pasture on the north side of the Sogamo- so opposite the house at Tablazo and no other geagraphic (sic) name for designating them was available in this area.

The shales of the Tablazo Pasture formation are lithologically very similar to those of the La Paja formation. They are thin bedded, finely laminated, black, in part, silty and slightly calcareous micaceous ahsles (sic) that slump on a large scale.

The Tablazo Pasture shales can be distingished (sic) from those of the La Paja in this area most readily by the abundance of small irregular shaped nodules and concretions of limonite and hematite that occur throught (sic) the formation. These nodules and concretions are usually less than 2" in their greatest dimension and around $1 / 2$ " or less in thickness. The absence of limestone concretions, septarians, and calcite veins and of worn (sic) tubes in these shales is an additional means of distinguishing them from those of the La Paja.

The limonite and hematite concretions are, most likely, epigenetic, in origin, derived by oxidation of pyrite above the water table. Below the water table, therefore, pyrite, rather than limonite and hematite would probably be present.

With the exception of a 4 " fossil redd (sic) of the upper part of the formation carrying many Exogyra and other fossils, the only fossils foune (sic) in these shales were ammonites. These seem to the (sic) fairly well distributed throughout the formation, algouth (sic) they were not particularly abundant.

Porous beds that might serve as a reservoir for oil are lacking in this formation.

The shales are expressed topographically by comparativerly (sic) low hills, often with abrupt edged (sic) due to slumping, that are difficult to ascend.

Debe notarse que la capa carrying many Exogyra mencionada de la parte superior del Tablazo Pasture Formation en la actualidad hace parte de la Formación El Salto (Terraza 2019).

\section{LOS AFLORAMIENTOS DE LA "FORMACIÓN" SIMITÍ EN EL SECTOR INVESTIGADO DE HIDROSOGAMOSO}

En el área del embalse Hidrosogamoso existen afloramientos discontinuos que no permiten describir de manera ininterrumpida la totalidad de la "Formación" Simití; hay exposiciones parciales de algunos tramos de la misma, así:

La parte inferior próxima al contacto con el techo de la Formación Tablazo aflora por la carretera nueva a San Vicente 
de Chucurí, cerca del sitio Guayacán (Lisboa). Se trata de lodolitas negras con muscovita que forman pendiente estructural dirigida hacia la carretera (288/54); presenta concreciones elipsoidales calcáreas, algunas de las cuales contienen restos de crustáceos, y están dispuestas de manera similar que los guijarros en las llanuras de mareas. Este tramo hoy en día está cubierto casi en su totalidad con pastos para protección de la carretera (fig. 2).

La parte media baja de la "Formación" Simití se mostraba en afloramiento al costado E de la carretera nueva a San Vicente de Chucurí que parte del sitio Lisboa hacia la quebrada La Molinilla (fig. 3). Se trata de lodolitas negras, físiles en superficie alterada (shales) con intercalaciones lenticulares entre decimétricas y centimétricas de calizas mudstone. Este tramo hoy en día presenta recubrimiento de protección.

La parte superior observable de la "Formación" Simití aflora en el costado oriental de la quebrada Aguablanca (fig. 4A). Allí se observa la sucesión invertida que se inicia (léase de arriba hacia abajo) al nivel del cauce de la quebrada con una caliza packstone que contiene cantos redondeados de caliza y concentraciones de conchas de bivalvos (tormentita proximal), y se apoya sobre un banco de cuarzoarenita de grano fino bioperturbada $(2,6 \mathrm{~m})$ con restos de amonitas (Ralphimlayites sp., fig. 4B); esta reposa sobre un intervalo de mudstone gris oscuro $(6 \mathrm{~m})$ que yace sobre un paquete de wackestone con amonitas (Mirapelia sp., fig. 4C), al cual, ascendiendo en la pared del afloramiento, le siguen 1,5 m de lodolita gris oscura que a su vez se apoya en otro banco $(70 \mathrm{~cm})$ de mudstone calcáreo seguido nuevamente por un tramo de lodolita gris oscura que remata en la cubierta del bosque (fig. 4A, Mapa geológico, G. Moreno-Sánchez 2019).

La "Formación" Simití aflora a trechos por el curso de la quebrada La Molinilla (véase Ward et al. 1973, 71 y Mapa geológico, G. Moreno-Sánchez 2019).

En las quebradas El Ramo y Pujamanes, así como en el río Sogamoso, no existen exposiciones continuas ni susceptibles de mediciones confiables de la unidad (véase Ward et al. 1973, 72 y Mapa geológico, G. Moreno-Sánchez 2019).

\section{BIOESTRATIGRAFÍA DE LA "FORMACIÓN" SIMITÍ CON BASE EN AMONITAS}

Maurice Breistroffer $(1952,2634)$ fue el primer paleontólogo que reconoció en Colombia el [...] Mesoalbien basal à Lyelliceras et Oxytropidoceras, específicamente Lyelliceras Ulrichi Knecht. En su discusión del Mésocrétacique en Europa y otras regiones $(1983,53)$, dicho autor señaló que en Le Mesoalbien Inferieur ou Hoplitien se presenta Oxytropidoceras du gr. Mirapelianum-roissyanum... Este punto de vista es adoptado por el autor del presente artículo (Etayo-Serna 1979, 14).

\section{REGIÓN DE HIDROSOGAMOSO}

Por la quebrada La Molinilla, en el tercio inferior de la "Formación" Simití se coleccionó Ralphimlayites ulrichi (KNECHTEL, 1947); esta amonita continúa apareciendo de modo intermitente hasta el tramo superior aflorante de la formación, en el cual se presenta además una capa con acumulación de Oxytropidoceras (Mirapelia) mirapelianum (ORBIGNY, 1850) (láms. 1/A, B y C; figs. 4A y 4B), que confirman el Albiano medio bajo.

\section{REGIÓN DE LA CIÉNAGA SIMITÍ}

Para precisar la edad de la "Formación" Simití en su sección tipo es necesario reconocer allí sus límites físicos. En el primer trabajo de dominio público conocido (Corredor 1955), se lee: Shale Simití. Estos shales que yacen en conformidad sobre las Calizas Aptianas tomaron su nombre de la población bolivarense de Simití, en donde se hallan especialmente bien expuestos. La designación es relativamente nueva, pues hasta hace poco tiempo se les llamó con el nombre provisional de shale-medio (middle shale) (Corredor 1955, 10; Petters 1954, 134, Middle Shale Formation).

Las Calizas Aptianas mencionadas de la ciénaga de Simití tienen cerca de $15 \mathrm{~m}$ de espesor, y en su aspecto macroscópico aparecen similares a las calizas de la Formación La Paja (Corredor 1955, columna estratigráfica y fotografías en la pág. 11). La edad aptiana de estas calizas se precisa con base en la presencia de Dufrenoyia codazziana (KARSTEN, 1858) y Dufrenoyia sanctorum BüRGL, 1956, según la revisión del presente autor del material paleontológico coleccionado por Corredor y conservado en la Facultad de Minas de la Universidad Nacional, sede Medellín.

En los alrededores de la cabecera municipal de Simití, de la formación homónima se coleccionaron además fósiles identificados como Douvilleiceras tarapacaense (ETAYo-SERNA, 1979) del Albiano inferior; Lyelliceras isaacleai (ETAYo-SERNA, 1979), Ralphimlayites alternum (ROBERT, 2002) y Ralphimlayites ulrichi (KNECHTEL, 1947) del Albiano medio bajo. Hacia la parte superior aflorante del shale de Simití se encontraron Hysteroceras bucklandi (SPATH, 1922) y Venezoliceras sp. del Albiano superior bajo; apenas $10 \mathrm{~m}$ estratigráficos encima de la muestra anterior están las calizas de la base de la Formación El Salto, de las cuales se obtuvo en las paredes de Ororia un ejemplar de Mantelliceras sp., del Cenomaniano inferior (Etayo-Serna en Ingeominas-Universidad Industrial de Santander 2006).

\section{LA "FORMACIÓN" SIMITÍ EN EL SUBSUELO}

En afloramientos del costado oriental del VMM se puede identificar con claridad la base de la "Formación" Simití y su relación estratigráfica con la Formación Tablazo. En sentido noroccidental, hacia la ciénaga de Simití, las calizas del Tablazo desaparecen gradualmente, como se descubre al com- 
parar los datos de subsuelo aportados por los pozos La Luna 1 (Juliao et al. 2013), Infantas 1613 (Taborda 1965, 1979, plate 1) y Llanito 1 (Cooper et al. 1995, fig. 4). Taborda ya había señalado (op. cit., 7), refiriéndose a la Tablazo Limestone, que "The formation is gradational with the Simití shale above, y aclaraba que In the subsurface, the Simití consists of shales and occasional thin beds of limestone and sandstone [...] These sandstone beds appear to grade into sandy limestone, which is brown and glauconitic" (ibid., 8).

\section{ESPESOR DE LA "FORMACIÓN" SIMITÍ}

En la región de la ciénaga de Simití los espesores atribuidos al Simití shale varían desde 550 m (Corredor 1995, 10), 400 $m$ (Morales y The Colombian Petroleum Industry 1958), hasta $40 \mathrm{~m}$ (Julivert 1968, lapsus calami). Del emblemático pozo Infantas 1613 se dice que mide 2.154 pies ( $665 \mathrm{~m})$, pero conviene recordar que esta cifra no representa el espesor estratigráfico, pues la perforación atravesó la unidad, cuya inclinación era mayor de $16^{\circ}$, como se ve en la lámina III, fig. II del Field Trip de Mares Concession (Hobson 1942; Taborda 1965, 1979). En la región de puente Tablazo se calcula para el Simití shale alrededor de $125 \mathrm{~m}$ (fig. 1), valor que contrasta con los 500 m que le atribuyó Wheeler (1929).

\section{ATISBO DE HISTORIA PALEOGEOGRÁFICA DE LA "FORMACIÓN" SIMITÍ}

A mediados del Albiano, tiempo durante el cual se acumularon los sedimentos de la "Formación" Simití, se produjo profundización del margen oriental del VMM, que había sufrido retracción a finales del depósito de la Formación Tablazo, durante el Albiano inicial tardío (Montoya 2019). Este mar inte- rior, de profundidad moderada (Cáceres, Cediel y Etayo-Serna 2005, fig. 8), ocupó posición ecuatorial-tropical (Moulin, Aslanian y Unternehr 2010, fig 25, Intra-Albiano), sus aguas fueron cálidas y en su fondo se acumuló un depósito finogranular muscovítico en el que a intervalos se produjeron condiciones ambientales que favorecieron el desarrollo de rasgos similares a los de la facies verdín moderna, caracterizada por la presencia de peloides verdosos verdine facies (Odin 1990) (Harding et al. 2014), que recuerdan los peloides que en la "Formación" Simití han sido identificados de modo tradicional como "glauconita" (Taborda 1965, 1979; figs. 5A y 5B).

\section{EL CONTACTO INCONFORME SIMITÍ-EL SALTO}

En la región de Hidrosogamoso, delgadas calizas de la Formación El Salto se apoyan sobre shale de la "Formación" Simití del Albiano medio bajo, mientras que en la región de la ciénaga de Simití, gruesas calizas de la Formación El Salto se apoyan sobre shales de la "Formación" Simití del Albiano superior bajo, de la misma manera como se observa esta relación de inconformidad entre la Formación San Gil Superior y la Formación Churuvita, en la región de Villa de Leiva (Etayo-Serna en Caicedo 2005, figs. 2-9).

El faltante bioestratigráfico Albiano medio alto-Albiano superior alto que se observa al comparar la bioestratigrafía de la "Formación" Simití del flanco SE (Hidrosogamoso) con la del flanco NW (ciénaga de Simití) del VMM sugiere un episodio de erosión (Etayo-Serna en Ingeominas-Universidad Industrial de Santander 2006), que determina una relación de disconformidad entre el techo del Simití y la base de El Salto. Nótese, además, que el equivalente de la Formación Tablazo en el VMM es la porción inferior de la "Formación" Simití del W del VMM.

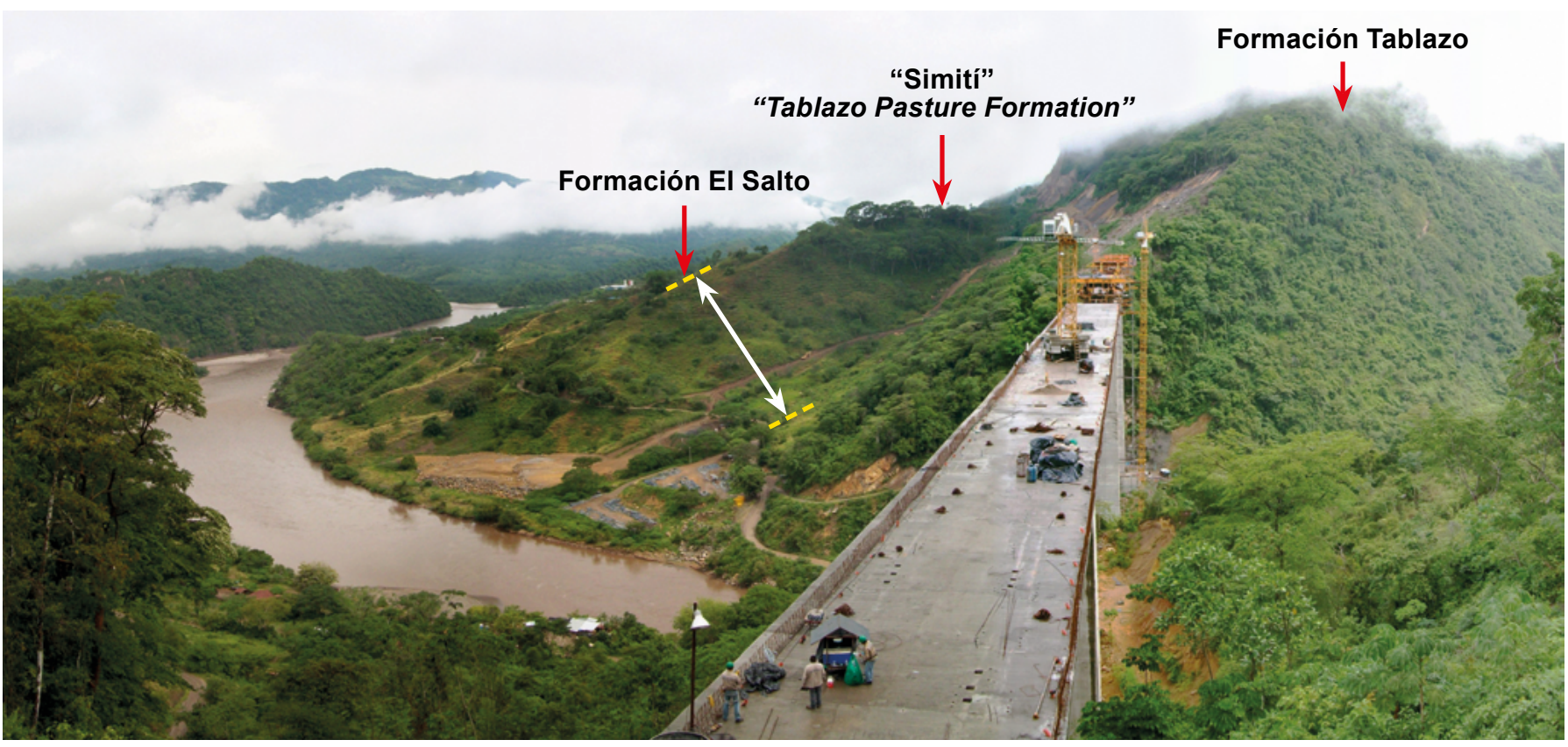

Figura 1. Localidad tipo de la Tablazo Pasture Formation de Wheeler (1929), hoy en día "Formación" Simití. Las líneas punteadas de color amarillo señalan los contactos con las formaciones Tablazo (inferior) y El Salto (superior). Vista desde un punto con coordenadas $\mathrm{N}: 1.269 .864, \mathrm{E}: 1.081 .171, \mathrm{Z}: 356 \mathrm{~m}$ (antes del llenado del embalse). Fotografía: G. Moreno-Sánchez. 


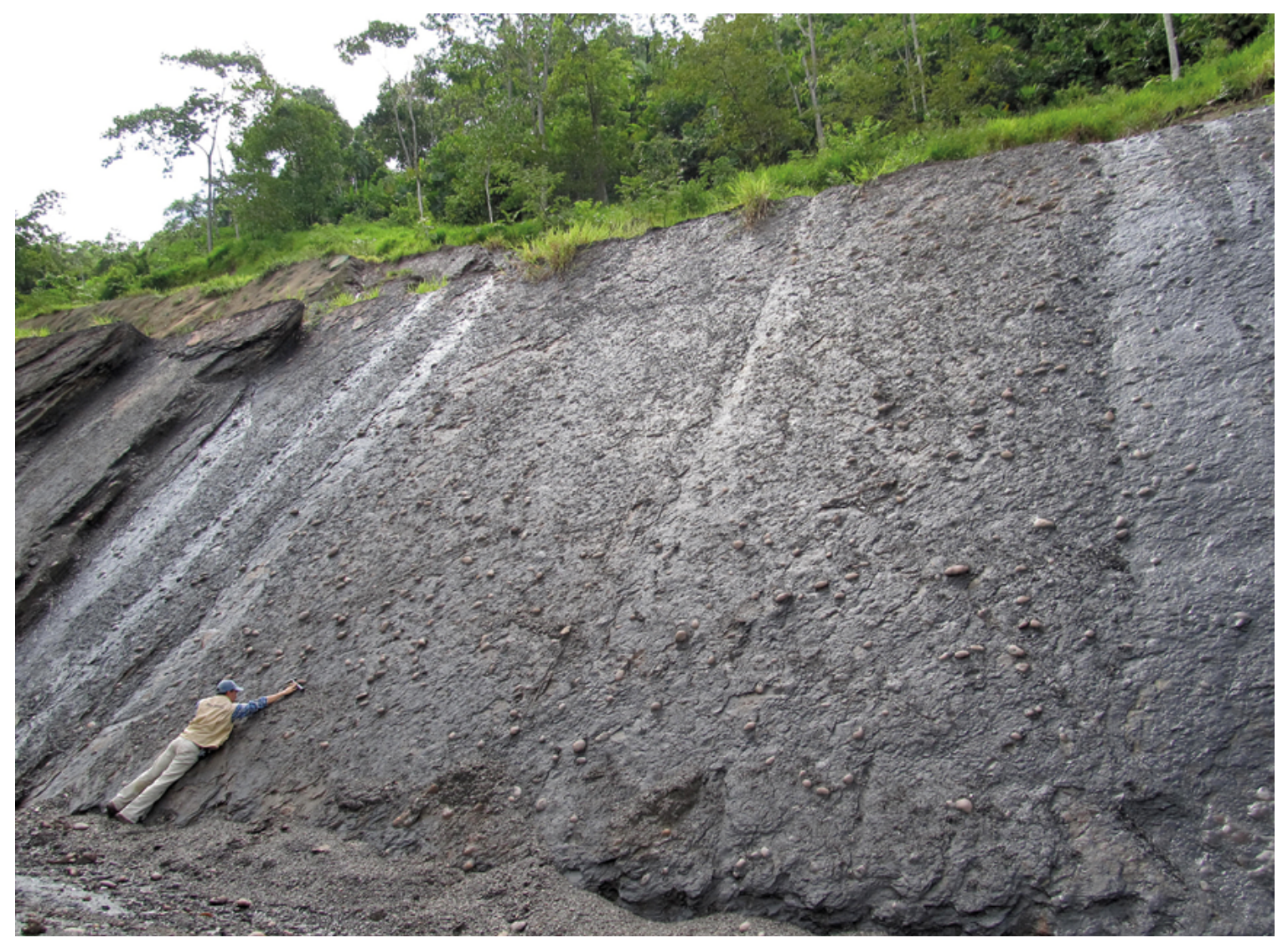

Figura 2. Afloramiento de la parte inferior de la "Formación" Simití por la carretera que rodea el embalse Hidrosogamoso y se dirige a San Vicente de Chucurí. N:1.281.300, E:1.085.878, Z:304. Fotografía: D. Montoya.

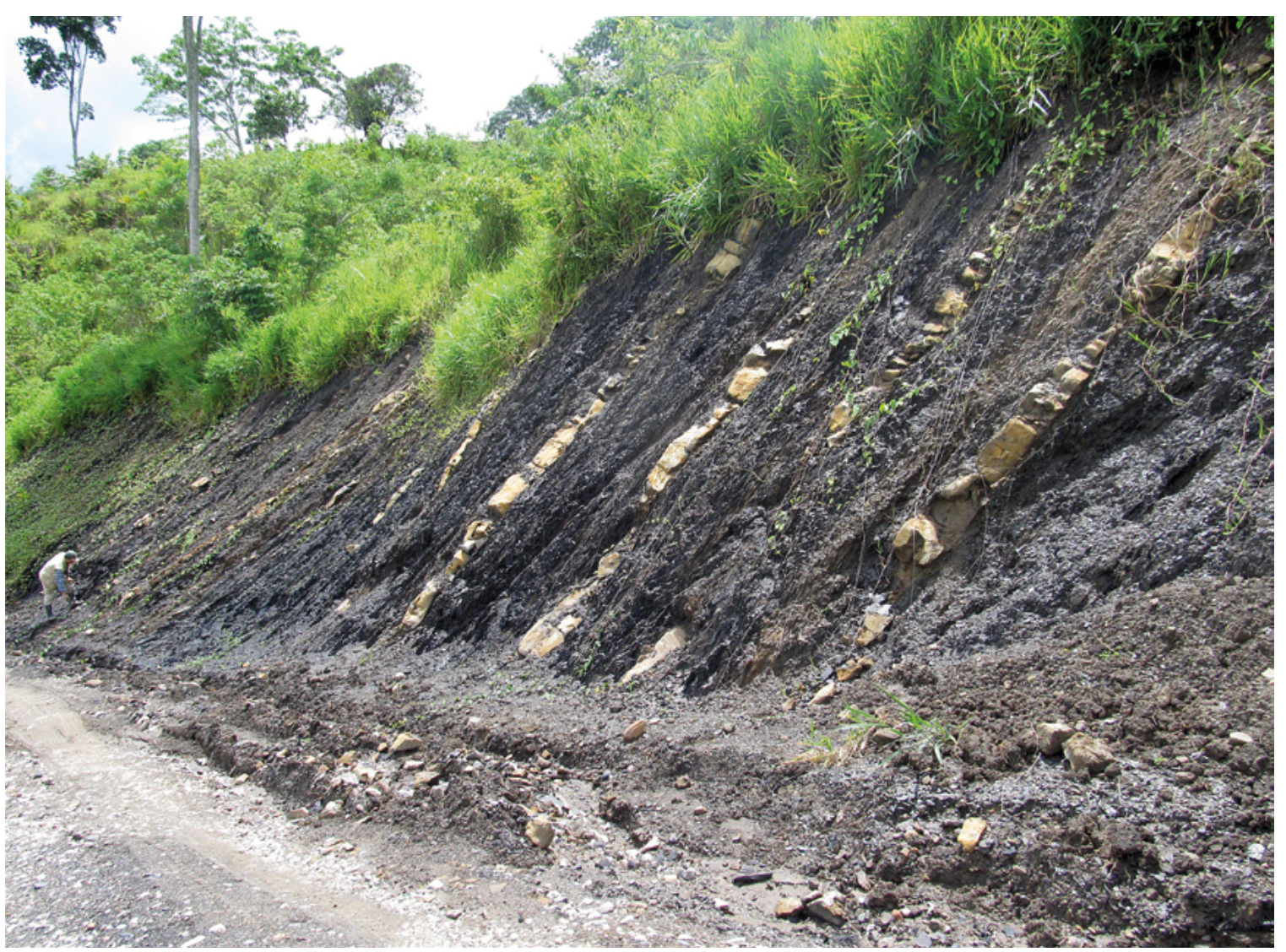

Figura 3. Parte media baja de la "Formación" Simití. Vía a San Vicente de Chucurí. N:1.280.526, E: 1.085.228, Z:632 m. Fotografía: D. Montoya. 


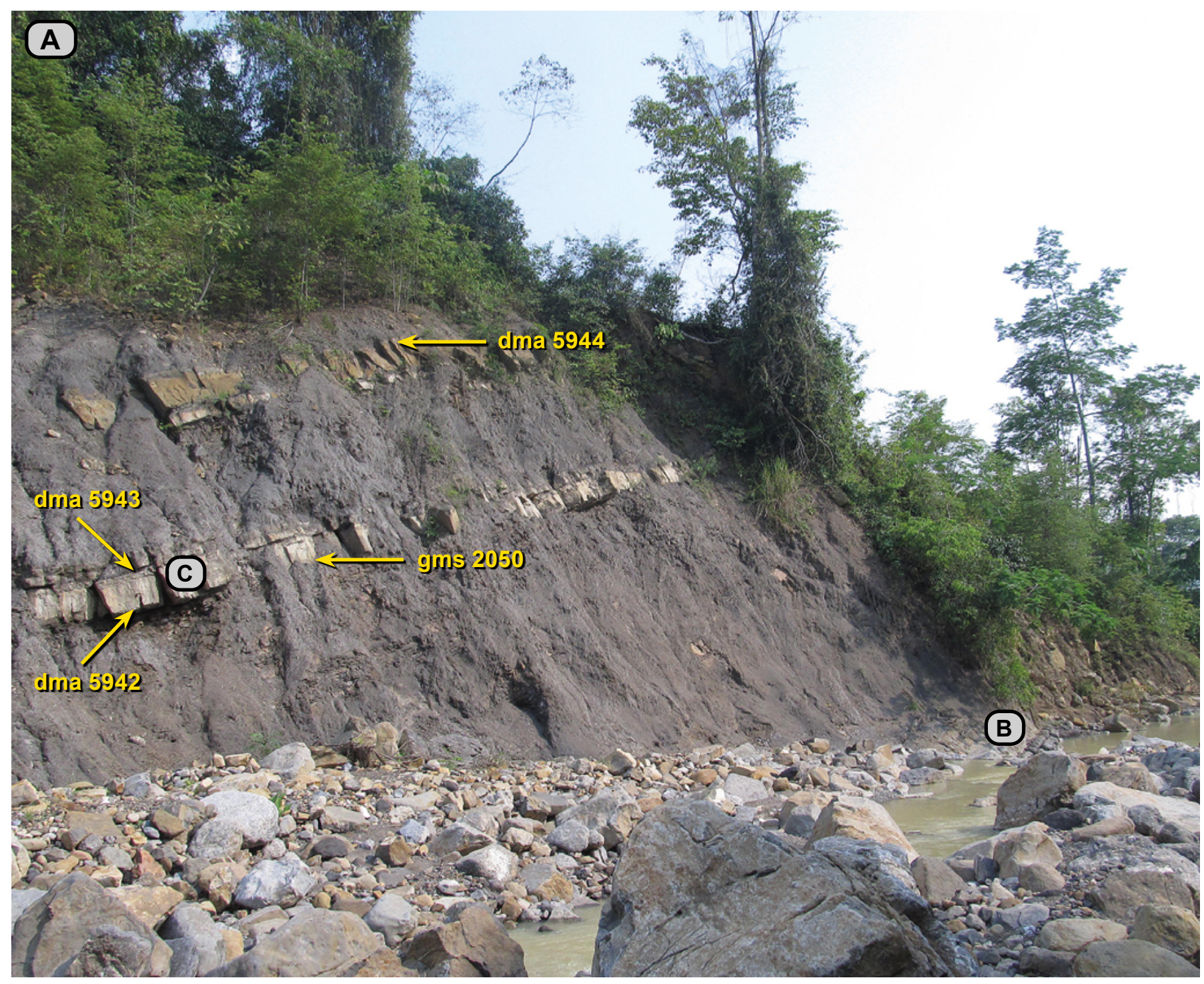

Figura 4A. Afloramiento de la parte superior de la "Formación" Simití, invertida en la margen oriental de la quebrada Aguablanca. $\mathrm{N}: 1.278 .123, \mathrm{E}: 1.083 .925, \mathrm{Z}: 322 \mathrm{~m}$. Buzamiento: N18E/54SE.

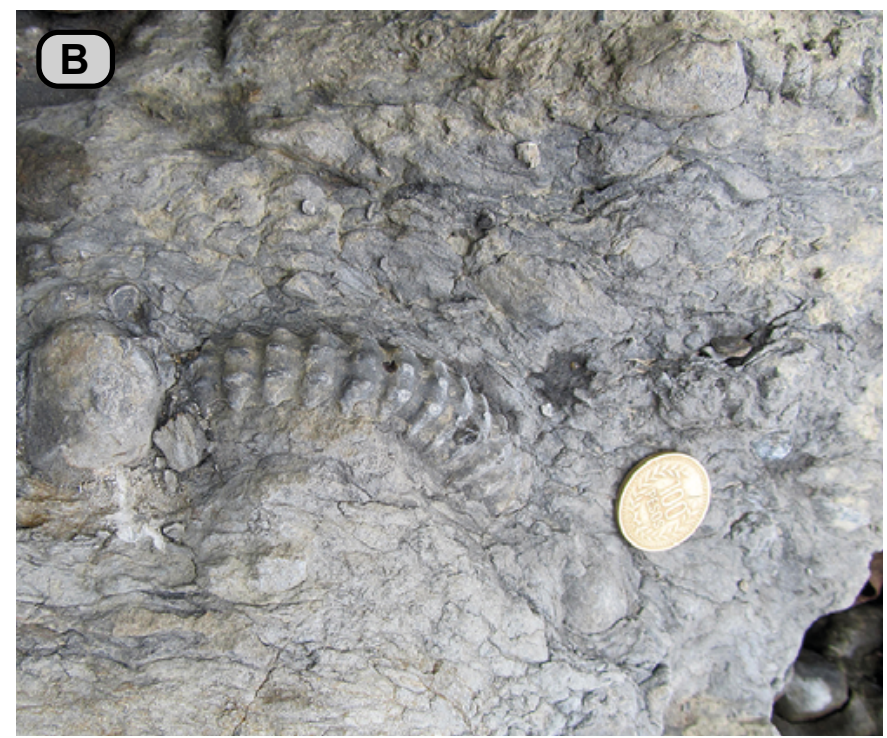

Figura 4B. Arenitas de cuarzo con cemento calcáreo bioturbadas, con Ralphimlayites sp. fig. 4A. Diámetro de la moneda: $22 \mathrm{~mm}$.

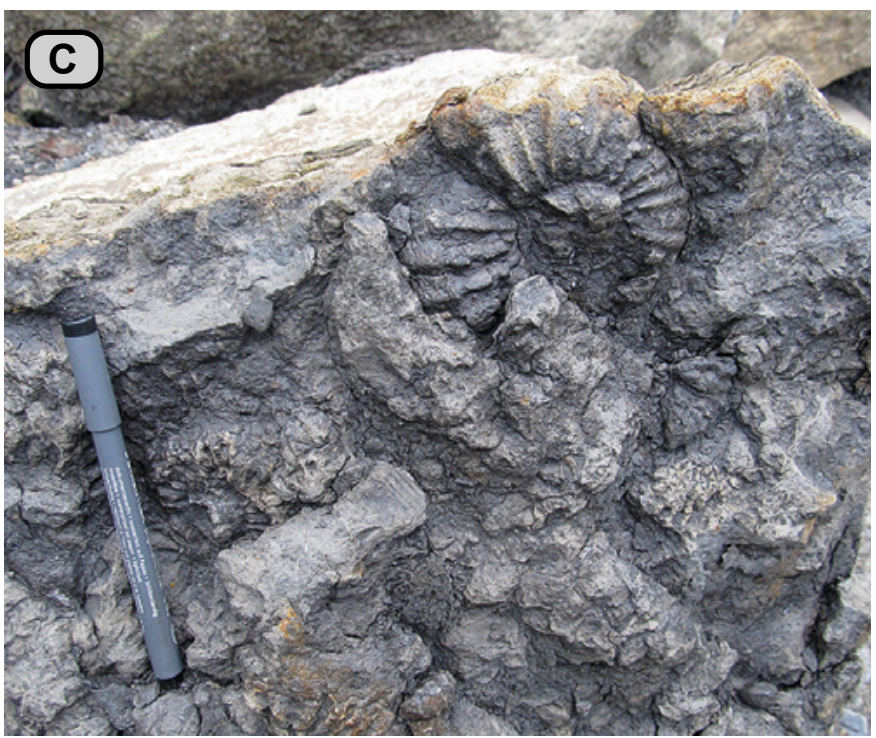

Figura 4C. Packstone con Oxytropidoceras (Mirapelia) sp. fig. 4A. Longitud del bolígrafo: $13,5 \mathrm{~cm}$. 

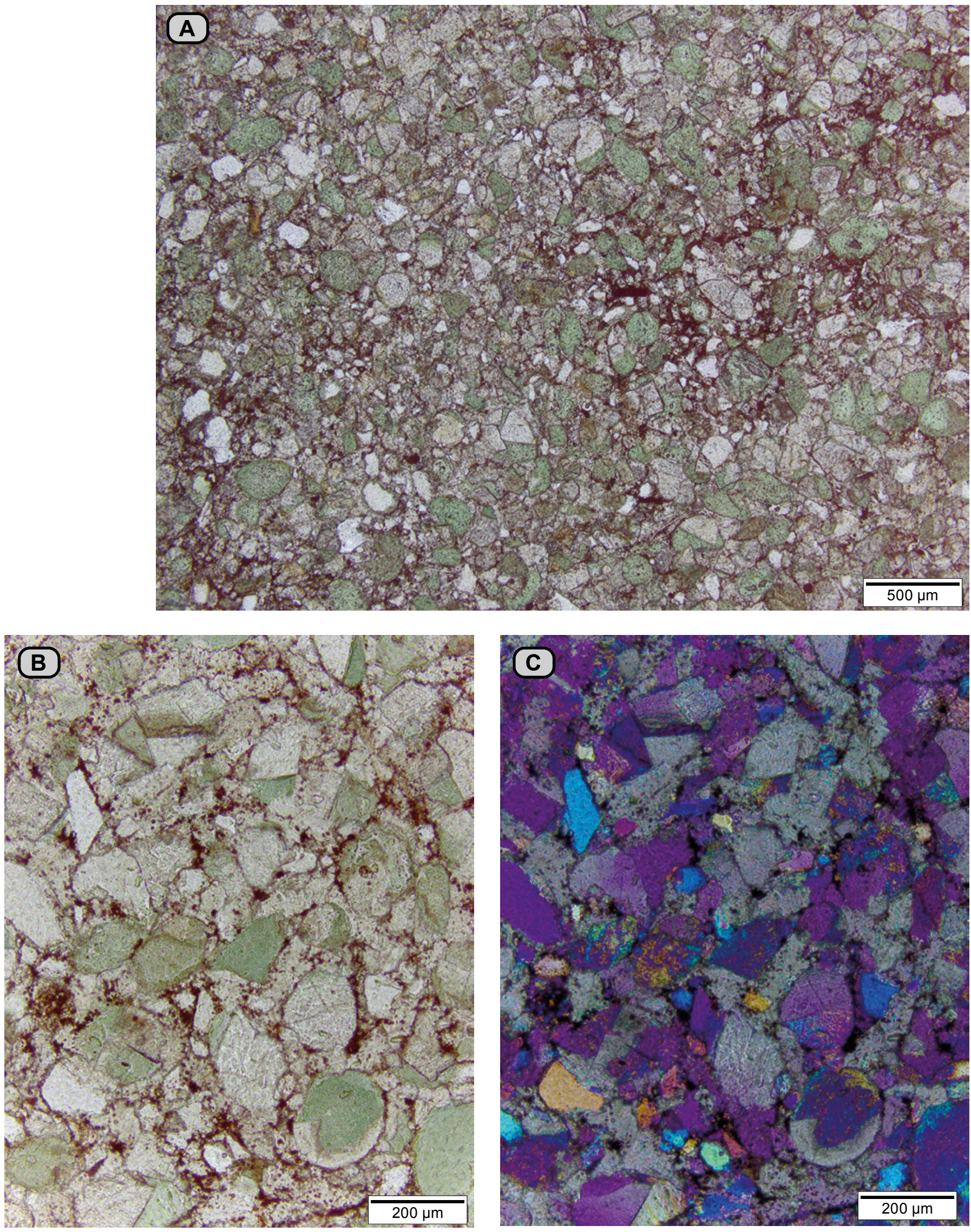

Figura 5. A) Aspecto general de la muestra dma 5944. Bajo el microscopio se reconocen los siguientes constituyentes: peloides de "glauconita" (41\%), cuarzo (10\%), cemento esparítico $37 \%$ y óxidos de hierro $(11 \%)$, de textura arena fina $(\mathrm{N}=)$. B) Detalle de la figura anterior (N=). C) (NX). Microfotografía: D. Montoya. 

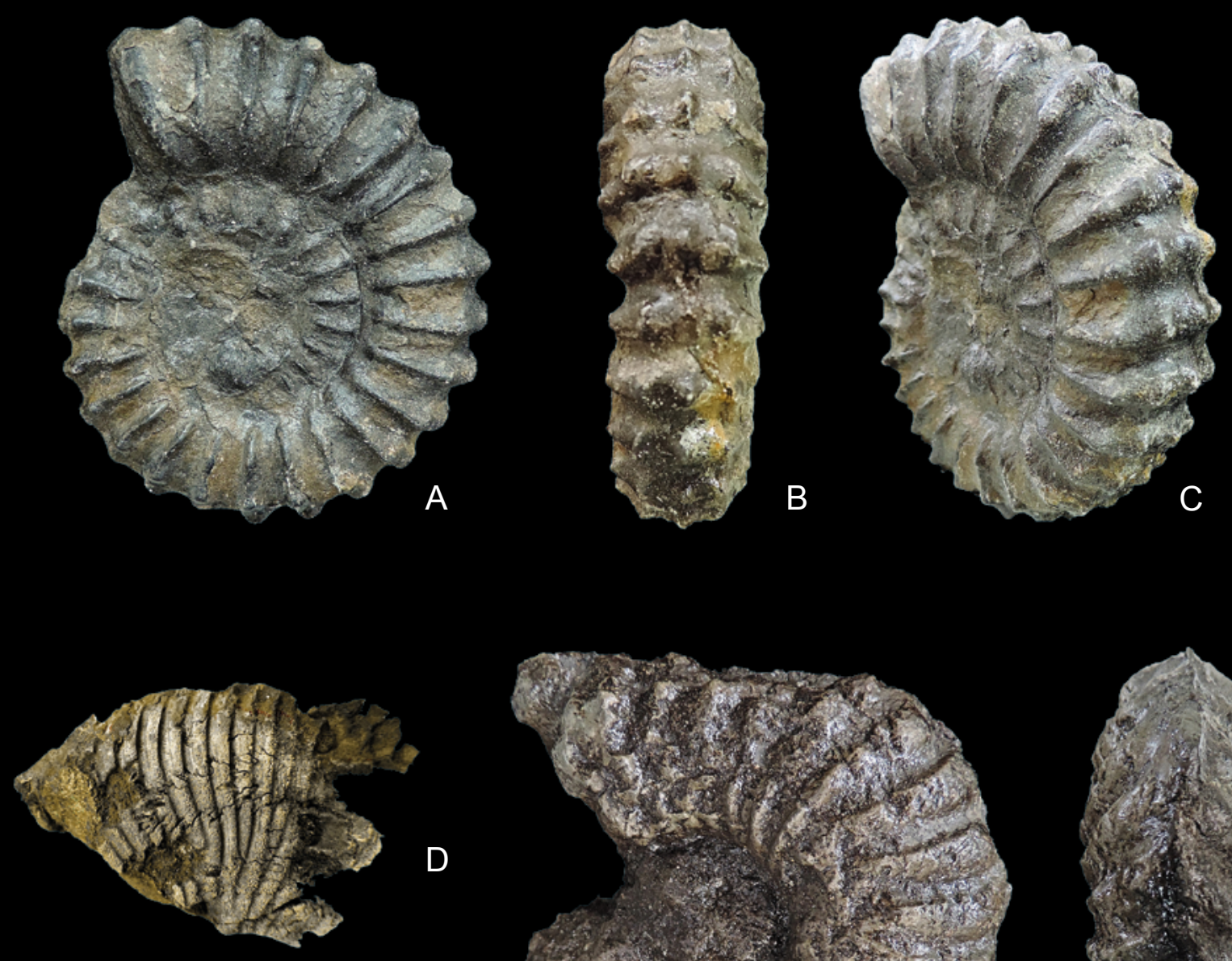

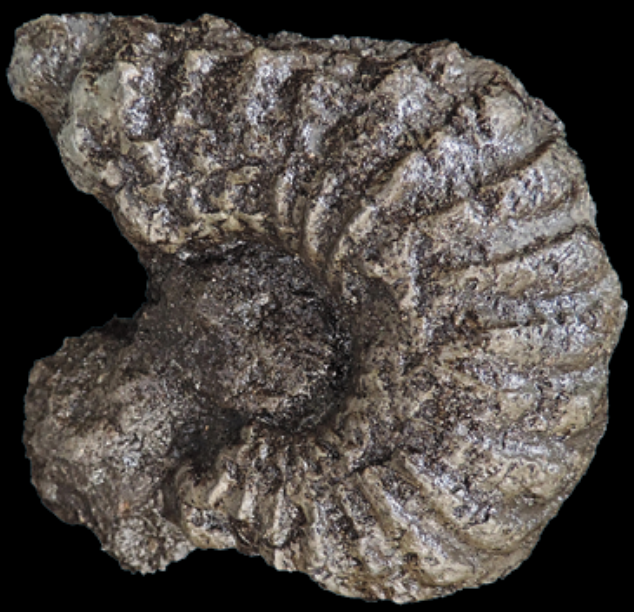

$\mathrm{E}$

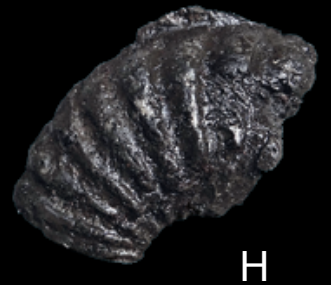

$\mathrm{H}$

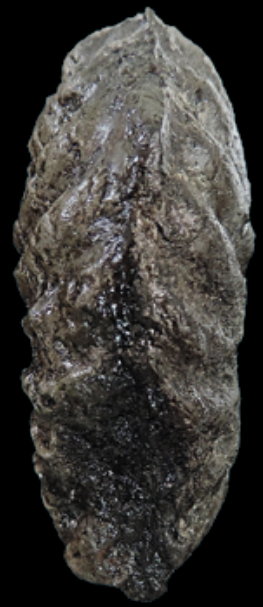

F
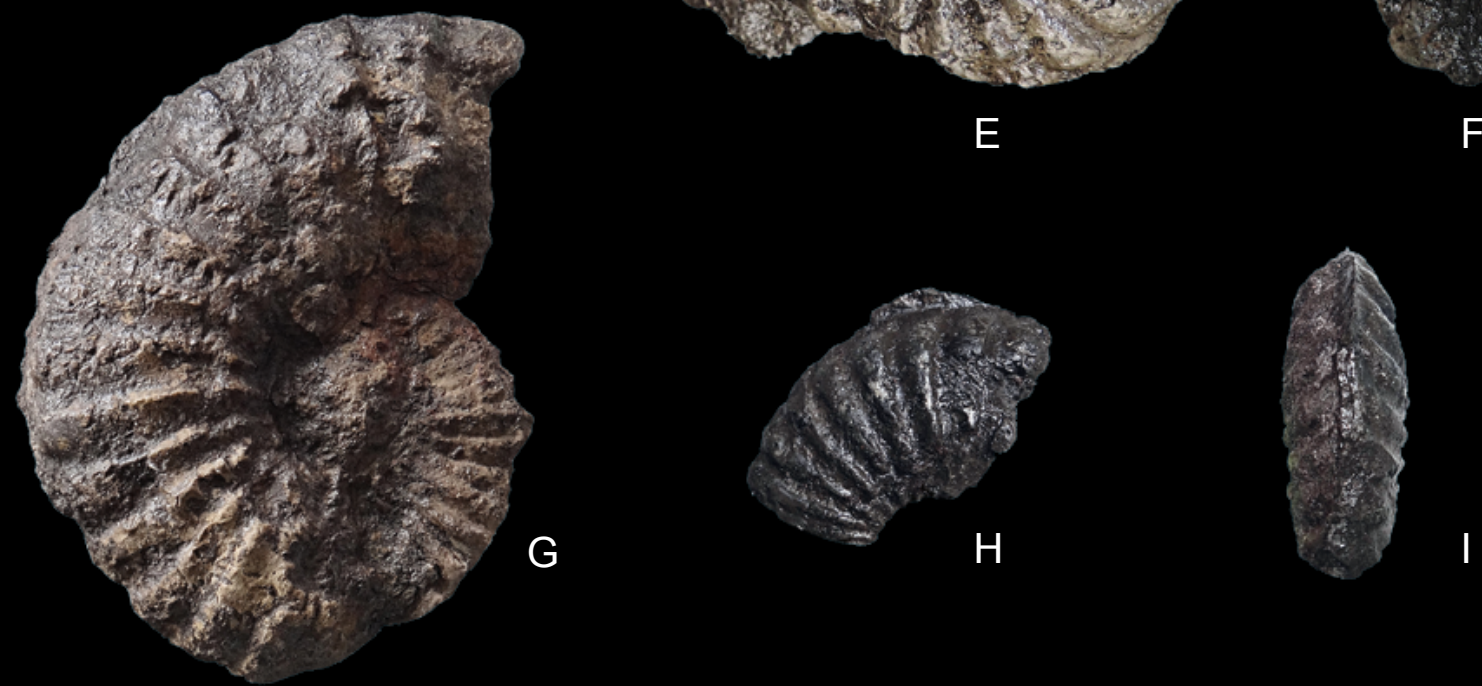

$1 \mathrm{~cm}$

Lámina 1. Ralphimlayites ulrichi (KNECHTEL, 1947). A, B y C: GMA 2070. Quebrada La Molinilla (N:1.279.708, E:1.084.912, Z:457) tercio inferior de la "Formación" Simití. Oxytropidoceras (Oxytropidoceras) hubbardi (KNECHTEL, 1947). D: dma 5938, tercio superior de la "Formación" Simití, Quebrada La Molinilla (N:1.279.869, E:1.084.633, Z:408). Oxytropidoceras (Mirapelia) mirapelianum (ORBIGNY, 1850). E, F, H, I: GMA 2050. G: dma 5942 (N:1.278.134, E:1.083994, Z:313) aproximadamente 20 m debajo del techo de la "Formación" Simití, quebrada Aguablanca. Las amonitas ilustradas representan el Albiano medio bajo. Fotografías: Alejandro Numpaque. 


\section{REFERENCIAS BIBLIOGRÁFICAS}

Ager, Derek V. 1993. The nature of the stratigraphical record. New York: John Wiley \& Sons Ltd.

Breistroffer, Maurice. 1952. "Sur la découverte de Knemiceratinae (Ammonites albiennes) en Équateur, en Colombie et au Vénézuela". Comptes Rendus hebdomadaires des séanses de L'Académie des Sciences de France 234 (27): 2633-2635. https:// gallica.bnf.fr/ark:/12148/bpt6k3186w?rk=21459;2.

Breistroffer, Maurice. 1983. "Le Mésocrétacique à ammonites en Europe et ailleurs". 108e Congrès national des Sociétés savantes. Sciences, fascicule 1, tome 2. Grenoble.

Cáceres Girón, Carlos, Fabio Cediel Melo y Fernando Etayo-Serna. 2005. Maps of sedimentary facies distribution and tectonic setting of Colombia through the Proterozoic and Phanerozoic. Bogotá: Ingeominas.

Caicedo Andrade, Juan Carlos. 2005. "Significado facial de la Formación Churuvita y su respuesta cartográfica". Tesis de maestria. Universidad Nacional de Colombia.

Cooper, Mark A., Fergus T. Addison, Ricardo Álvarez, Mario Coral, Rod H. Graham, Anthony B. Hayward, Spencer Howe, Jaime Martínez, Joaquín Naar, Ricardo Peñas, Andy J. Pulham y Adriana Taborda. 1995. "Basin development and tectonic history of the Llanos Basin, Eastern Cordillera, and Middle Magdalena Valley, Colombia”. AAPG Bulletin 79 (10): 1421-1443.

Corredor, Ernesto. 1955. "Apuntes sobre la geología de la región de Simití, departamento de Bolívar". Tesis de grado. Universidad Nacional de Colombia, Sede Medellín.

Etayo-Serna, Fernando. 1979. Zonation of the Cretaceous of central Colombia by ammonites. Publicaciones geológicas especiales 2. Bogotá: Ingeominas.

Harding, Sherie C., Barbara P. Nash, Erich U. Petersen, A. A. Ekdale, Christopher D. Bradbury y M. Darby Dyar M. 2014. "Mineralogy and geochemistry of the main glauconite bed in the Middle Eocene of Texas: Paleoenvironmental implications for the verdine facies". PloS ONE 9 (2): e87656. https://doi:or/10.137/journal.pone.0087656.

Hobson, Geoffrey Dudley. 1942. "Calculating the true thickness of a folded bed: Geological Notes". Bulletin of the American Association of Petroleum Geologists 26 (12): 1827-1832. https:// doi.org/10.1306/3D933578-16B1-11D7-8645000102C1865D.

Hubach, Enrique. 1957. “Contribución a las unidades estratigráficas de Colombia (enumeración regional, de más reciente a más antiguo)". Informe 1212. Bogotá: Instituto Geológico Nacional.

Ingeominas-Universidad Industrial de Santander. 2006. "Cartografía geológica de $9.600 \mathrm{~km}^{2}$ de la serranía de San Lucas. Planchas 55 (El Banco), 64 (Barranco de Loba), 85 (Simití) y 96 (Bocas de Rosario). Aporte al conocimiento de su evolución geológica”. Tomo 1. "Memoria explicativa, plancha 85 Simití, sur de los departamentos de Bolívar y Cesar". Tomo 5. "Catálogo de sedimentos activos y suelos, catálogo de petrografía, catálogo paleontológico y resultados de inclusiones fluidas". "Catálogo paleontológico de la plancha 85" por Fernando Etayo-Serna: 28 páginas sin numeración. Bogotá: Servicio Geológico Colombiano.

Juliao, Tatiana Milena, Marcelo de Araujo Carvalho, Sandra Milena Cruz y Robert Emilio Márquez. 2013. "Reconstrucción paleoambiental basada en palinofacies y geoquímica orgánica para las formaciones La Luna y Tablazo en el pozo La Luna 1, Cuenca VMM". XVI Congreso Colombiano de Geología. Primer Simposio de Exploradores.

Julivert, Manuel. 1968. "Colombie (première partie), Précambrien, Paléozoïque, et intrusions d'âge mésozoïque-tertiaire". En Lexique
Stratigraphique International V. Amérique Latine, dirigido por R. Hoffstetter, fascicule 4a. Paris: Centre National De La Recherche Scientifique.

Montoya Arenas, Diana M. 2019. “Formación Tablazo: descripción de la sección tipo. Depósitos de carbonatos en una rampa afectada por exposiciones subaéreas iterativas". En Estudios geológicos y paleontológicos sobre el Cretácico en la región del embalse del río Sogamoso, Valle Medio del Magdalena, dirección científica y edición de Fernando Etayo-Serna. Compilación de los Estudios Geológicos Oficiales en Colombia vol. XXIII. Bogotá: Servicio Geológico Colombiano.

Morales, Luis G. y The Colombian Petroleum Industry. 1958. “General geology and oil occurrences of Middle Magdalena Valley, Colombia". In Habitat of oil. A symposium; conducted by the American Association of Petroleum Geologists, edited by Lewis G. Weeks, 641-695. Special Publication 18. Tulsa: The American Association of Petroleum Geologists.

Moreno-Sánchez, Giovanni. 2019. “Mapa geológico del Valle Medio del Magdalena en los alrededores del puente El Tablazo, departamento de Santander". En Estudios geológicos y paleontológicos sobre el Cretácico en la región del embalse del río Sogamoso, Valle Medio del Magdalena, dirección científica y edición de Fernando EtayoSerna. Compilación de los Estudios Geológicos Oficiales en Colombia vol. XXIII. Bogotá: Servicio Geológico Colombiano.

Moulin, Maryline, Daniel Aslanian y Patrick Unternehr. 2010. "A new starting point for the South and Equatorial Atlantic 0cean". EarthScience Reviews 98 (1-2): 1-37. https://doi.org/10.1016/j.earscirev.2009.08.001.

Odin, Gilles Serge. 1990. "Clay mineral formation at the continentocean boundary: the verdine facies". Clay Minerals 25 (4): 477483. https://doi.org/10.1180/claymin.1990.025.4.06.

Petters, Victor. 1954. "Typical foraminiferal horizons in the Lower Cretaceous of Colombia, S. A.". Contributions from the Cushman Foundation for Foraminiferal Research 5 (3): 128-137. https://cushmanfoundation.allenpress.com/portals/_default/files/pubarchive/CCFFR/05ccffr3.pdf.

Salvador, Amos. 1994. International stratigraphic guide: A guide to stratigraphic classification, terminology, and procedure. Boulder: The International Union of Geological Sciences y The Geological Society of America, Inc.

Taborda, Bernardo. 1965. Guidebook to the geology of the De Mares Concession: field trip of February 26-28, 1965. Bogotá: Colombian Society of Petroleum Geologists and Geophysicists.

Taborda, Bernardo. 1979. "The geology of the Mares Concession. Fifth Field Conference. February 26-28, 1965". En Geological FieldTrips Colombia 1959-1978. Bogotá: Colombian Society of Petroleum Geologists and Geophysicists.

Terraza Melo, Roberto. 2019. “'Formación La Luna': expresión espuria en la geología colombiana”. En Estudios geológicos y paleontológicos sobre el Cretácico en la región del embalse del río Sogamoso, Valle Medio del Magdalena, dirección científica y edición de Fernando Etayo-Serna. Compilación de los Estudios Geológicos Oficiales en Colombia vol. XXIII. Bogotá: Servicio Geológico Colombiano.

Ward, Dwight E., Richard Goldsmith, Jaime Cruz y Hernán Restrepo A. 1973. "Geología de los cuadrángulos H-12 Bucaramanga y H-13 Pamplona, departamento de Santander”. Boletín Geológico 21 (13): $1-134$.

Wheeler, Orby Clinton. 1929. "Report on the Palmira Series with Notes on Stratigraphy of the Umir, Lisama, and La Paz Formations near the Eastern part of the Mares Concession". Informe Geológico 37. Ecopetrol. 